\title{
Figure Legends
}

Fig. 1: Conditions of auditory and visual stimulation. Time courses for the three conditions of adaptation (Scatter, Adaptation Same, Adaptation Opposite) are shown as position vs. time diagrams. The phases of stimulation (adaptation, stationary phase, test motion) are separated by dotted lines. The sequence was repeated in a cyclic design within different blocks for each stimulus condition. Adaptation was always visual, while the stationary phase plus test stimuli were either visual (A) or auditory (gray background, B).

Fig. 2: Time courses of the blocks measured in each session. Each block was preceded by a 2min motion adaptation phase. Here, 105 repetitions of the motion adaptation stimulus that was also used for top-up adaptation during the adaptation phase of the recordings were presented, with 500-ms silence between each of the adaptation stimuli. For the recordings, 115 trials comprising top-up adaptation and tests were presented consecutively (see Fig. 1 for details). Pre-adaptation and top-up adaptation was always visual, while the stationary phase plus test stimuli were either visual (vis-vis) or auditory (vis-aud, grayed out). The order of the blocks varied between subjects.

Fig. 3. Hit rates (\%), i.e., the detection of test stimuli moving in the direction opposite to adaptation, for every condition and test stimulus (mean \pm SEM; $n=21$ ). There were no significant effects of adaptation type (Adaptation Same, Scatter, Adaptation Opposite) or test stimulus types (auditory or visual) on the performance.

Fig. 4: Topography of grand mean motion-onset ERPs for the auditory test stimuli. Motion onset, i.e., beginning of the test phase at time $0 \mathrm{~ms}$, is indicated by the vertical line; $\mathrm{n}=21$. The typical response complex comprising $\mathrm{cN} 1$ and $\mathrm{cP} 2$ is evident at fronto-central sites. Greek symbols $(\Sigma, \Phi)$ mark the electrodes identified in the cluster analysis.

Fig. 5: Motion-onset ERPs to auditory test stimuli identified in the cluster analysis. (A, C) Grand mean traces for Scatter, Adaptation-Opposite, and Adaptation-Same conditions ( $\mathrm{n}=$ 21) for the group sites identified in the cluster analysis (corresponding electrodes are shown in the head view). Conditions depicted in solid lines were compared in the cluster analysis; conditions in dashed lines are only shown for comparison. Motion onset of the test phase is indicated by the solid vertical line. The cluster range is indicated by the black bar between vertical dashed lines. Asterisks indicate the significance level determined in the single-peak 
analysis. (B) cP1-amplitudes (mean $\pm \mathrm{SEM} ; \mathrm{n}=21$ ) for Scatter and Adaptation Opposite as determined with a single peak analysis for each individual for the group site $(\Phi: F C 3, F C 1$, $\mathrm{FCz}, \mathrm{C} 3, \mathrm{C} 1, \mathrm{CP} 1)$. The $\mathrm{P}$-value $(\mathrm{P}=0.0022)$ was obtained in a paired t-test. (D) cP1amplitudes (mean \pm SEM; $\mathrm{n}=21$ ) for Scatter and Adaptation Same as determined with a single peak analysis for each individual for the group site ( $\Sigma$ : F4, FC3, FC1, FCz, FC2, FC4, $\mathrm{C} 3, \mathrm{C} 1, \mathrm{Cz}, \mathrm{C} 2)$. The $\mathrm{P}$-value $(\mathrm{P}=0.013)$ was obtained in a paired t-test.

Fig. 6: Topography of grand mean motion-onset ERPs for the visual test stimuli $(\mathrm{n}=21)$. Conventions as for Figure 4. Greek symbols $(\Psi, \Omega, \Pi)$ mark the electrodes according to the cluster analysis.

Fig. 7: Motion-onset ERPs to visual test stimuli identified in the cluster analysis. (A, C, E) Grand mean traces for Scatter, Adaptation Opposite, and Adaptation Same $(n=21)$ for the group sites identified in the cluster analysis. Conventions as for Figure 5. (B) cP1, (D) cP2, and (F) cN2 amplitudes (mean \pm SEM; $n=21$ ) are shown for Adaptation-Same (AdaSame) and Adaptation-Opposite (AdaOpp) conditions, as determined with a single peak analysis for each individual for the group site (cP1, $\Psi$ : FC4, FC3, Cz, C2, CP2, P4, PO4; cP2, $\Omega$ : F4, FC1, $\mathrm{FCz}, \mathrm{FC} 2, \mathrm{FC} 4, \mathrm{Cz}, \mathrm{C} 2$; cN2, П: P8, PO8, O2, Oz). The P-values were obtained in a paired ttest.

Fig. 8. Motion-onset ERPs to visual test stimuli - linked mastoids reference. (A) Grand mean traces $(n=21)$ for Scatter, Adaptation-Opposite, and Adaptation-Same conditions for the electrodes $\mathrm{Cz}, \mathrm{PO}$, Oz, PO8, referenced to linked mastoids. Asterisks indicate the significance level determined in the single-peak analysis. (B) $\mathrm{cP} 2(\mathrm{Cz})$ and cN2-amplitudes (PO7, Oz, PO8; mean \pm SEM; $\mathrm{n}=21$ ) for Scatter, Adaptation Opposite (AdaOpp), and Adaptation Same (AdaSame), as determined with a single peak analysis for each individual electrode. The P-values were obtained in paired t-tests and corrected for multiple testing. 